\title{
AN ERGODIC THEOREM FOR ASYMPTOTICALLY NONEXPANSIVE MAPPINGS IN THE INTERMEDIATE SENSE
}

\author{
HIROKAZU OKA
}

(Communicated by Palle E. T. Jorgensen)

\begin{abstract}
This paper is concerned with an ergodic theorem for asymptotically nonexpansive mappings in the intermediate sense in Banach spaces.
\end{abstract}

\section{INTRODUCTION AND THE MAIN RESULT}

Throughout this paper $X$ denotes a uniformly convex real Banach space, $C$ a nonempty closed convex subset of $X$, and $T$ a mapping from $C$ into itself.

Recently the asymptotic behavior of asymptotically nonexpansive mappings has been studied by many authors (see [4], [14], [15] and [16]). There appear in the literature two definitions of an asymptotically nonexpansive mapping. One is due to Kirk [6] :

$$
\limsup _{n \rightarrow \infty} \sup _{y \in K}\left(\left\|T^{n} x-T^{n} y\right\|-\|x-y\|\right) \leq 0
$$

for each $x \in C$ and each bounded set $K \subset C$, and $T^{N}$ is continuous for some $N \geq 1$. The other is due to Goebel and Kirk [5] : There exists a sequence $\left\{k_{n}\right\}$ with $\lim _{n \rightarrow \infty} k_{n}=1$ such that

$$
\left\|T^{n} x-T^{n} y\right\| \leq k_{n}\|x-y\| \text { for } x, y \in C \text { and } n=0,1,2, \cdots .
$$

Bruck, Kuczumow and Reich [4] have introduced a definition between these two: $T$ is called asymptotically nonexpansive in the intermediate sense if $T$ is continuous and

$$
\limsup _{n \rightarrow \infty} \sup _{x, y \in K}\left(\left\|T^{n} x-T^{n} y\right\|-\|x-y\|\right) \leq 0
$$

for any bounded subset $K \subset C$. The purpose of the present paper is to prove the ergodic theorem for such a mapping in the class of Banach spaces in which the nonlinear mean ergodic theorem is usually set.

Here we summarize the notations used in the sequel. Denote by $F(T)$ the set of fixed points of a mapping $T$. The convex hull of a subset $M$ of $X$ is denoted by

Received by the editors May 4, 1995 and, in revised form, November 7, 1995 and December 5, 1995.

1991 Mathematics Subject Classification. Primary 47H09, 47H10.

Key words and phrases. Asymptotically nonexpansive mapping in the intermediate sense, almost-orbit, weak almost convergence, convex approximation property.

(C)1997 American Mathematical Society 
$c o M$, and the closed convex hull by $c l c o M$. We put

$$
\Delta^{n-1}=\left\{\lambda=\left(\lambda_{1}, \cdots, \lambda_{n}\right): \lambda_{i} \geq 0(i=1,2, \cdots, n) \text { and } \sum_{i=1}^{n} \lambda_{i}=1\right\}
$$

and

$$
B_{r}=\{x \in X:\|x\| \leq r\} \text { for } r>0 .
$$

Also, $\omega_{w}\left(\left\{z_{n}\right\}\right)$ denotes the set of weak subsequential limits of a sequence $\left\{z_{n}\right\}_{n \geq 0}$ in $X$.

Definition 1.1. A sequence $\left\{x_{n}\right\}_{n \geq 0}$ in $C$ is called an almost-orbit of $T$ if

$$
\lim _{n \rightarrow \infty}\left[\sup _{m \geq 0}\left\|x_{n+m}-T^{m} x_{n}\right\|\right]=0 .
$$

Definition 1.2. A sequence $\left\{z_{n}\right\}_{n \geq 0}$ in $X$ is said to be weakly almost convergent to $z \in X$ if $\frac{1}{n} \sum_{i=0}^{n-1} z_{i+k}$ converges weakly as $n \rightarrow \infty$ to $z$ uniformly in $k \geq 0$.

The main result in this paper is stated as follows:

Theorem 1.1. Suppose that $T: C \rightarrow C$ is asymptotically nonexpansive in the intermediate sense with $F(T) \neq \phi$, and that $\left\{x_{n}\right\}$ is an almost-orbit of $T$. If the norm of $X$ is Fréchet differentiable, then $\left\{x_{n}\right\}$ is weakly almost convergent to the unique point of $F(T) \cap \operatorname{clcow}_{w}\left(\left\{x_{n}\right\}\right)$.

\section{Proof of theorem}

In what follows, a mapping $T: C \rightarrow C$ is assumed to be asymptotically nonexpansive in the intermediate sense with $F(T) \neq \phi$. Take $f \in F(T)$ and let $K$ be a bounded closed convex subset of $C$ including the set $\{f\}$. Put $D_{K}=\operatorname{diameter} K$.

The key point in proving mean ergodic theorems is to estimate the difference between $T^{k}\left(\sum_{i=1}^{n} \lambda_{i} z_{i}\right)$ and $\sum_{i=1}^{n} \lambda_{i} T^{k} z_{i}$ for $\lambda \in \Delta^{n-1}, z_{1}, \cdots, z_{n} \in K$ and $k \geq 1$, as done previously in [15, Proposition 3.1] and [8, Lemma 3]. However, we cannot make use of Bruck's inequality [3, Theorem 2.1] as used in [15] and [8], because our operator $T$ is not Lipschitz continuous. Therefore our argument is different from theirs.

Lemma 2.1. For $\varepsilon>0$ there exist an integer $N_{\varepsilon} \geq 1$ and $\delta_{2, \varepsilon}>0$ such that if $k \geq N_{\varepsilon}, z_{1}, z_{2} \in K$ and if $\left\|z_{1}-z_{2}\right\|-\left\|T^{k} z_{1}-T^{k} z_{2}\right\| \leq \delta_{2, \varepsilon}$, then

$$
\left\|T^{k}\left(\lambda_{1} z_{1}+\lambda_{2} z_{2}\right)-\lambda_{1} T^{k} z_{1}-\lambda_{2} T^{k} z_{2}\right\|<\varepsilon
$$

for all $\lambda=\left(\lambda_{1}, \lambda_{2}\right) \in \Delta^{1}$.

Proof. Let $\delta$ be the modulus of uniform convexity of $X$ and define a function $d$ : $\mathbf{R}^{+} \rightarrow \mathbf{R}^{+}$by

$$
d(t)= \begin{cases}\frac{1}{2} \int_{0}^{t} \delta(s) d s & \text { if } 0 \leq t \leq 2 \\ d(2)+\frac{1}{2} \delta(2)(t-2) & \text { if } t>2\end{cases}
$$

It is then well known that $d$ is a strictly increasing, continuous convex function, and that it satisfies

$$
2 \lambda_{1} \lambda_{2} d(\|u-v\|) \leq 1-\left\|\lambda_{1} u+\lambda_{2} v\right\|
$$

for $\lambda=\left(\lambda_{1}, \lambda_{2}\right) \in \Delta^{1},\|u\| \leq 1$ and $\|v\| \leq 1$. 
For $\varepsilon>0$ choose $\eta_{\varepsilon}>0$ such that $\frac{D_{K}}{2} d^{-1}\left(\frac{2 \eta_{\varepsilon}}{D_{K}}\right)<\varepsilon$ and put $\delta_{2, \varepsilon}=\min \left\{\eta_{\varepsilon}, \frac{D_{K}}{4}\right\}$. By (1.2) there exists an integer $N_{\varepsilon} \geq 1$ (depending on the set $K$ ) such that if $k \geq N_{\varepsilon}$,

$$
\left\|T^{k} x-T^{k} y\right\|-\|x-y\|<\delta_{2, \varepsilon} \text { for all } x, y \in K .
$$

Let $k \geq N_{\varepsilon}$ and let $z_{1}, z_{2} \in K$ with $\left\|z_{1}-z_{2}\right\|-\left\|T^{k} z_{1}-T^{k} z_{2}\right\| \leq \delta_{2, \varepsilon}$. It suffices to show Lemma 2.1 in the case of $0<\lambda_{i}<1(i=1,2)$.

Put

$$
u=\frac{T^{k} z_{2}-T^{k}\left(\lambda_{1} z_{1}+\lambda_{2} z_{2}\right)}{\lambda_{1}\left(\left\|z_{1}-z_{2}\right\|+\delta_{2, \varepsilon}\right)} \text { and } v=\frac{T^{k}\left(\lambda_{1} z_{1}+\lambda_{2} z_{2}\right)-T^{k} z_{1}}{\lambda_{2}\left(\left\|z_{1}-z_{2}\right\|+\delta_{2, \varepsilon}\right)} .
$$

Then we have $\|u\| \leq 1,\|v\| \leq 1$ and

$$
\lambda_{1} u+\lambda_{2} v=\frac{T^{k} z_{2}-T^{k} z_{1}}{\left\|z_{1}-z_{2}\right\|+\delta_{2, \varepsilon}} .
$$

Since

$$
u-v=\frac{\lambda_{1} T^{k} z_{1}+\lambda_{2} T^{k} z_{2}-T^{k}\left(\lambda_{1} z_{1}+\lambda_{2} z_{2}\right)}{\lambda_{1} \lambda_{2}\left(\left\|z_{1}-z_{2}\right\|+\delta_{2, \varepsilon}\right)}
$$

and $\frac{2}{D_{K}} \lambda_{1} \lambda_{2}\left(\left\|z_{1}-z_{2}\right\|+\delta_{2, \varepsilon}\right) \leq \frac{2}{D_{K}} \cdot \frac{1}{4} \cdot\left(D_{K}+\frac{D_{K}}{4}\right)<1$, we have by (2.1) and $(2.2)$

$$
\begin{aligned}
& d\left(\frac{2}{D_{K}}\left\|\lambda_{1} T^{k} z_{1}+\lambda_{2} T^{k} z_{2}-T^{k}\left(\lambda_{1} z_{1}+\lambda_{2} z_{2}\right)\right\|\right) \\
& \leq \frac{2}{D_{K}} \lambda_{1} \lambda_{2}\left(\left\|z_{1}-z_{2}\right\|+\delta_{2, \varepsilon}\right) d(\|u-v\|) \\
& \leq \frac{2}{D_{K}} \lambda_{1} \lambda_{2}\left(\left\|z_{1}-z_{2}\right\|+\delta_{2, \varepsilon}\right) \frac{1}{2 \lambda_{1} \lambda_{2}}\left\{1-\frac{\left\|T^{k} z_{1}-T^{k} z_{2}\right\|}{\left\|z_{1}-z_{2}\right\|+\delta_{2, \varepsilon}}\right\} \\
& =\frac{1}{D_{K}}\left(\left\|z_{1}-z_{2}\right\|-\left\|T^{k} z_{1}-T^{k} z_{2}\right\|+\delta_{2, \varepsilon}\right) \leq \frac{2 \delta_{2, \varepsilon}}{D_{K}} \leq \frac{2 \eta_{\varepsilon}}{D_{K}} .
\end{aligned}
$$

Here we have used the convexity of a function $d$ and the fact that $d(0)=0$. Consequently, we obtain from the choice of $\eta_{\varepsilon}$

$$
\left\|T^{k}\left(\lambda_{1} z_{1}+\lambda_{2} z_{2}\right)-\lambda_{1} T^{k} z_{1}-\lambda_{2} T^{k} z_{2}\right\| \leq \frac{D_{K}}{2} d^{-1}\left(\frac{2 \eta_{\varepsilon}}{D_{K}}\right)<\varepsilon .
$$

Lemma 2.2. For each $\varepsilon>0$ and each integer $n \geq 2$ there exist an integer $N_{\varepsilon} \geq 1$ and $\delta_{n, \varepsilon}>0$, where $N_{\varepsilon}$ is independent of $n$, such that if $k \geq N_{\varepsilon}, z_{1}, \cdots, z_{n} \in K$ and if $\left\|z_{i}-z_{j}\right\|-\left\|T^{k} z_{i}-T^{k} z_{j}\right\| \leq \delta_{n, \varepsilon}$ for $1 \leq i, j \leq n$, then

$$
\left\|T^{k}\left(\sum_{i=1}^{n} \lambda_{i} z_{i}\right)-\sum_{i=1}^{n} \lambda_{i} T^{k} z_{i}\right\|<\varepsilon
$$

for all $\lambda=\left(\lambda_{1}, \cdots, \lambda_{n}\right) \in \Delta^{n-1}$.

Proof. Let $\varepsilon>0$ and let $n \geq 2$ be an arbitrary integer. Choose an integer $N_{\varepsilon} \geq 1$ in Lemma 2.1. We shall construct $\delta_{n, \varepsilon}(n=2,3, \cdots)$ inductively. Let $\delta_{2, \varepsilon}$ be as in Lemma 2.1. Suppose that all $\delta_{q, \varepsilon}$ are constructed for $q=2, \cdots, p$. Let $\varepsilon^{\prime}=\min \left(\frac{1}{3} \delta_{p, \frac{\varepsilon}{2}}, \frac{\varepsilon}{2}\right)$ and put $\delta_{p+1, \varepsilon}=\min \left(\delta_{2, \varepsilon^{\prime}}, \varepsilon^{\prime}\right)$. 
Let $\lambda \in \Delta^{p}, z_{1}, \cdots, z_{p+1} \in K, k \geq N_{\varepsilon}$ and $\left\|z_{i}-z_{j}\right\|-\left\|T^{k} z_{i}-T^{k} z_{j}\right\| \leq \delta_{p+1, \varepsilon}$ for $1 \leq i, j \leq p+1$. The case $\lambda_{p+1}=1$ is trivial and so we assume $\lambda_{p+1} \neq 1$. Putting

$$
\begin{aligned}
& u_{j}=\left(1-\lambda_{p+1}\right) z_{j}+\lambda_{p+1} z_{p+1}, \mu_{j}=\frac{\lambda_{j}}{1-\lambda_{p+1}} \text { and } \\
& u_{j}^{\prime}=\left(1-\lambda_{p+1}\right) T^{k} z_{j}+\lambda_{p+1} T^{k} z_{p+1}
\end{aligned}
$$

for $j=1,2, \cdots, p$, we have

$$
\begin{aligned}
& \sum_{i=1}^{p+1} \lambda_{i} z_{i}=\sum_{j=1}^{p} \frac{\lambda_{j}}{1-\lambda_{p+1}}\left\{\left(1-\lambda_{p+1}\right) z_{j}+\lambda_{p+1} z_{p+1}\right\}=\sum_{j=1}^{p} \mu_{j} u_{j} \text { and } \\
& \sum_{i=1}^{p+1} \lambda_{i} T^{k} z_{i}=\sum_{j=1}^{p} \mu_{j} u_{j}^{\prime}
\end{aligned}
$$

and hence

$$
\begin{aligned}
& \left\|T^{k}\left(\sum_{i=1}^{p+1} \lambda_{i} z_{i}\right)-\sum_{i=1}^{p+1} \lambda_{i} T^{k} z_{i}\right\|=\left\|T^{k}\left(\sum_{j=1}^{p} \mu_{j} u_{j}\right)-\sum_{j=1}^{p} \mu_{j} u_{j}^{\prime}\right\| \\
& \leq\left\|T^{k}\left(\sum_{j=1}^{p} \mu_{j} u_{j}\right)-\sum_{j=1}^{p} \mu_{j} T^{k} u_{j}\right\|+\sum_{j=1}^{p} \mu_{j}\left\|T^{k} u_{j}-u_{j}^{\prime}\right\| .
\end{aligned}
$$

Since $\left\|z_{j}-z_{p+1}\right\|-\left\|T^{k} z_{j}-T^{k} z_{p+1}\right\| \leq \delta_{p+1, \varepsilon} \leq \delta_{2, \varepsilon^{\prime}}$, we have by Lemma 2.1

$\left\|u_{j}^{\prime}-T^{k} u_{j}\right\|=\left\|\left\{\left(1-\lambda_{p+1}\right) T^{k} z_{j}+\lambda_{p+1} T^{k} z_{p+1}\right\}-T^{k}\left\{\left(1-\lambda_{p+1}\right) z_{j}+\lambda_{p+1} z_{p+1}\right\}\right\| \leq \varepsilon^{\prime}$ for $1 \leq j \leq p$ and

$$
\begin{aligned}
\left\|u_{j}-u_{l}\right\|-\left\|u_{j}^{\prime}-u_{l}^{\prime}\right\| & =\left(1-\lambda_{p+1}\right)\left\{\left\|z_{j}-z_{l}\right\|-\left\|T^{k} z_{j}-T^{k} z_{l}\right\|\right\} \\
& \leq\left(1-\lambda_{p+1}\right) \delta_{p+1, \varepsilon} \leq \varepsilon^{\prime}=\min \left(\frac{1}{3} \delta_{p, \frac{\varepsilon}{2}}, \frac{\varepsilon}{2}\right)
\end{aligned}
$$

for $1 \leq j, l \leq p$. Therefore we obtain

$$
\begin{aligned}
\left\|u_{j}-u_{l}\right\|-\left\|T^{k} u_{j}-T^{k} u_{l}\right\| & \leq\left\|u_{j}-u_{l}\right\|-\left\|u_{j}^{\prime}-u_{l}^{\prime}\right\|+\left\|u_{l}^{\prime}-T^{k} u_{l}\right\|+\left\|u_{j}^{\prime}-T^{k} u_{j}\right\| \\
& \leq \frac{1}{3} \delta_{p, \frac{\varepsilon}{2}}+\varepsilon^{\prime}+\varepsilon^{\prime} \leq \delta_{p, \frac{\varepsilon}{2}}
\end{aligned}
$$

for $1 \leq j, l \leq p$, and thus by the inductive assumption and (2.3) the desired conclusion holds.

Since $X$ is uniformly convex, it has the convex approximation property (C.A.P.), i.e. for each $\varepsilon>0$ there exists an integer $p(=p(\varepsilon)) \geq 1$ such that for all subsets $M$ in $X$ whose diameters are uniformly bounded,

$$
c o M \subset c o_{p} M+B_{\varepsilon},
$$

where $c o_{p} M:=\left\{\lambda_{1} z_{1}+\cdots+\lambda_{p} z_{p}: \lambda \in \Delta^{p-1}, z_{1}, \cdots, z_{p} \in M\right\}$ (see [3, Theorem $1.1])$.

The following lemma shows that the positive number $\delta_{n, \varepsilon}$ in Lemma 2.2 can be chosen independently of $n$, thanks to this property of the space $X$. 
Lemma 2.3. For every $\varepsilon>0$ and every integer $n \geq 2$ there exist an integer $N_{\varepsilon} \geq 1$ and $\delta_{\varepsilon}>0$, where both $N_{\varepsilon}$ and $\delta_{\varepsilon}$ are independent of $n$, such that if $k \geq N_{\varepsilon}$, $z_{1}, \cdots, z_{n} \in K$ and if $\left\|z_{i}-z_{j}\right\|-\left\|T^{k} z_{i}-T^{k} z_{j}\right\| \leq \delta_{\varepsilon}$ for $1 \leq i, j \leq n$, then

$$
\left\|T^{k}\left(\sum_{i=1}^{n} \lambda_{i} z_{i}\right)-\sum_{i=1}^{n} \lambda_{i} T^{k} z_{i}\right\|<\varepsilon
$$

for all $\lambda \in \Delta^{n-1}$.

Proof. Fix $\varepsilon>0$ and an integer $n \geq 2$ arbitrarily. Denote by $N_{1, \varepsilon}$ the integer $N_{\frac{\varepsilon}{4}}$ in Lemma 2.2. By (1.2) there is an integer $N_{2, \varepsilon} \geq 1$ such that if $k \geq N_{2, \varepsilon}$, then we have

$$
\left\|T^{k} x-T^{k} y\right\|-\|x-y\|<\varepsilon / 4 \text { for all } x, y \in K .
$$

Put $N_{\varepsilon}=\max \left(N_{1, \varepsilon}, N_{2, \varepsilon}\right)$. Let $\delta_{n, \varepsilon}(n=2,3, \cdots)$ be positive numbers determined in Lemma 2.2. As pointed out in the proof of [3, Theorem 2.1], $X \times X$ has the C.A.P. and hence we can choose an integer $p(=p(\varepsilon)) \geq 1$ such that

$$
c o M \subset c o_{p} M+B_{\varepsilon / 4} \times B_{\varepsilon / 4}
$$

for all subsets $M$ in $X \times X$ whose diameters are uniformly bounded. Note that this integer $p$ is independent of $n$. Put $\delta_{\varepsilon}=\delta_{p, \frac{\varepsilon}{4}}$.

Let $k \geq N_{\varepsilon}, z_{1}, \cdots, z_{n} \in K$, and $\left\|z_{i}-z_{j}\right\|-\left\|T^{k} z_{i}-T^{k} z_{j}\right\| \leq \delta_{\varepsilon}(1 \leq i, j \leq n)$. Consider $M=\left\{\left[z_{i}, T^{k} z_{i}\right] \in X \times X: i=1,2, \cdots, n\right\}$. Note that there exists $r>0$, independent of $k$ and $n$, such that $\sup _{(x, y) \in M}\|(x, y)\|_{X \times X} \leq r$ because of (2.5) and $f \in F(T) \cap K$. Then for each $\lambda \in \Delta^{n-1}$ there exist $\mu \in \Delta^{p-1}$ and $i_{1}, \cdots, i_{p} \in\{1,2, \cdots, n\}$ such that

$$
\left\|\sum_{i=1}^{n} \lambda_{i} z_{i}-\sum_{j=1}^{p} \mu_{j} z_{i_{j}}\right\|<\varepsilon / 4
$$

and

$$
\left\|\sum_{i=1}^{n} \lambda_{i} T^{k} z_{i}-\sum_{j=1}^{p} \mu_{j} T^{k} z_{i_{j}}\right\|<\varepsilon / 4 .
$$

Therefore we have by (2.5) and the choice of $\delta_{\varepsilon}$

$$
\begin{aligned}
& \left\|T^{k}\left(\sum_{i=1}^{n} \lambda_{i} z_{i}\right)-\sum_{i=1}^{n} \lambda_{i} T^{k} z_{i}\right\| \\
& \leq\left\|T^{k}\left(\sum_{i=1}^{n} \lambda_{i} z_{i}\right)-T^{k}\left(\sum_{j=1}^{p} \mu_{j} z_{i_{j}}\right)\right\|+\left\|T^{k}\left(\sum_{j=1}^{p} \mu_{j} z_{i_{j}}\right)-\sum_{j=1}^{p} \mu_{j} T^{k} z_{i_{j}}\right\| \\
& +\left\|\sum_{j=1}^{p} \mu_{j} T^{k} z_{i_{j}}-\sum_{i=1}^{n} \lambda_{i} T^{k} z_{i}\right\|<\varepsilon .
\end{aligned}
$$

For each $\varepsilon>0$ and each integer $k \geq 1$ set

$$
F_{\varepsilon}\left(T^{k}\right)=\left\{x \in C:\left\|T^{k} x-x\right\| \leq \varepsilon\right\} .
$$


Lemma 2.4. For each $\varepsilon>0$ there exist an integer $N(\varepsilon) \geq 1$ and $\delta(=\delta(\varepsilon))>0$ such that

$$
\operatorname{clco}\left(F_{\delta}\left(T^{k}\right) \cap K\right) \subset F_{\varepsilon}\left(T^{k}\right) \cap K
$$

for all $k \geq N(\varepsilon)$.

Proof. For each $\varepsilon>0$ choose an integer $N_{\frac{\varepsilon}{2}} \geq 1$ and $\delta_{\frac{\varepsilon}{2}}>0$ in Lemma 2.3.

Let $k \geq N(\varepsilon):=N_{\frac{\varepsilon}{2}}$ and put $\delta:=(\delta(\varepsilon)=) \min \left(\frac{1}{2} \delta_{\frac{\varepsilon}{2}}, \frac{\varepsilon}{2}\right)$. Let $y \in \operatorname{co}\left(F_{\delta}\left(T^{k}\right) \cap K\right)$, say $y=\sum_{i=1}^{n} \beta_{i} y_{i}\left(y_{i} \in F_{\delta}\left(T^{k}\right) \cap K, i=1,2, \cdots, n ; \beta \in \Delta^{n-1}\right)$. By Lemma 2.3,

$$
\begin{aligned}
& \left\|T^{k}\left(\sum_{i=1}^{n} \beta_{i} y_{i}\right)-\sum_{i=1}^{n} \beta_{i} y_{i}\right\| \\
& \leq\left\|T^{k}\left(\sum_{i=1}^{n} \beta_{i} y_{i}\right)-\sum_{i=1}^{n} \beta_{i} T^{k} y_{i}\right\|+\sum_{i=1}^{n} \beta_{i}\left\|T^{k} y_{i}-y_{i}\right\| \leq \varepsilon,
\end{aligned}
$$

because $\left\|y_{i}-y_{j}\right\|-\left\|T^{k} y_{i}-T^{k} y_{j}\right\| \leq\left\|y_{i}-T^{k} y_{i}\right\|+\left\|y_{j}-T^{k} y_{j}\right\| \leq 2 \delta \leq \delta_{\frac{\varepsilon}{2}}$ for $1 \leq i, j \leq n$. Therefore $c o\left(F_{\delta}\left(T^{k}\right) \cap K\right) \subset F_{\varepsilon}\left(T^{k}\right)$, which implies (2.6).

Lemma 2.5. Let $\left\{z_{n}\right\}$ be a sequence in $K$ such that $\mathrm{w}-\lim _{n \rightarrow \infty} z_{n}=z$. Suppose that for each $\varepsilon>0$ there exists an integer $N(\varepsilon) \geq 1$ such that for $k \geq N(\varepsilon)$ there is an integer $N_{k, \varepsilon} \geq 1$ satisfying $\left\|T^{k} z_{n}-z_{n}\right\|<\varepsilon$ for all $n \geq N_{k, \varepsilon}$. Then we have $z \in F(T)$.

Proof. We shall show that $\lim _{k \rightarrow \infty}\left\|T^{k} z-z\right\|=0$. For $\varepsilon>0$ choose an integer $N_{\frac{\varepsilon}{5}} \geq 1$ and $\delta_{\frac{\varepsilon}{5}}>0$ in Lemma 2.3. By (1.2) there exists an integer $N_{1}(\varepsilon) \geq 1$ such that if $k \geq N_{1}(\varepsilon)$,

$$
\left\|T^{k} u-T^{k} v\right\|-\|u-v\|<\frac{\varepsilon}{5}
$$

for all $u, v \in K$. Put $\varepsilon^{\prime}=\min \left(\frac{1}{2} \delta_{\frac{\varepsilon}{5}}, \frac{\varepsilon}{5}\right)$. By assumption we can take an integer $N\left(\varepsilon^{\prime}\right) \geq 1$. Let $N_{2}(\varepsilon):=\max \left(N_{\frac{\varepsilon}{5}}, N_{1}(\varepsilon), N\left(\varepsilon^{\prime}\right)\right)$ and let $k \geq N_{2}(\varepsilon)$.

Since $z \in \operatorname{clco}\left\{z_{n}: n \geq N_{k, \varepsilon^{\prime}}\right\}$, where $N_{k, \varepsilon^{\prime}}$ is the integer determined by assumption, there exists a sequence $\left\{\sum_{i=1}^{l_{n}} \lambda_{n}^{(i)} z_{\phi_{n}(i)}\right\} \subset \operatorname{co}\left\{z_{n}: n \geq N_{k, \varepsilon^{\prime}}\right\}$ such that $\lim _{n \rightarrow \infty} \sum_{i=1}^{l_{n}} \lambda_{n}^{(i)} z_{\phi_{n}(i)}=z$.

Since

$$
\begin{aligned}
& \left\|z_{\phi_{n}(i)}-z_{\phi_{n}(j)}\right\|-\left\|T^{k} z_{\phi_{n}(i)}-T^{k} z_{\phi_{n}(j)}\right\| \\
& \quad \leq\left\|z_{\phi_{n}(i)}-T^{k} z_{\phi_{n}(i)}\right\|+\left\|z_{\phi_{n}(j)}-T^{k} z_{\phi_{n}(j)}\right\| \leq \delta_{\frac{\varepsilon}{5}}\left(1 \leq i, j \leq l_{n}\right)
\end{aligned}
$$

by assumption, Lemma 2.3 implies

$$
\left\|T^{k}\left(\sum_{i=1}^{l_{n}} \lambda_{n}^{(i)} z_{\phi_{n}(i)}\right)-\sum_{i=1}^{l_{n}} \lambda_{n}^{(i)} T^{k} z_{\phi_{n}(i)}\right\|<\frac{\varepsilon}{5} .
$$

There is also $N_{3}(k, \varepsilon) \geq 1$ such that

$$
\left\|\sum_{i=1}^{l_{n}} \lambda_{n}^{(i)} z_{\phi_{n}(i)}-z\right\|<\frac{\varepsilon}{5}
$$


for all $n \geq N_{3}(k, \varepsilon)$. Since $z \in K$, the combination of the above inequalities with (2.7) gives

$$
\begin{aligned}
\left\|T^{k} z-z\right\| & \leq\left\|T^{k} z-T^{k}\left(\sum_{i=1}^{l_{n}} \lambda_{n}^{(i)} z_{\phi_{n}(i)}\right)\right\| \\
& +\left\|T^{k}\left(\sum_{i=1}^{l_{n}} \lambda_{n}^{(i)} z_{\phi_{n}(i)}\right)-\sum_{i=1}^{l_{n}} \lambda_{n}^{(i)} T^{k} z_{\phi_{n}(i)}\right\| \\
& +\left\|\sum_{i=1}^{l_{n}} \lambda_{n}^{(i)}\left(T^{k} z_{\phi_{n}(i)}-z_{\phi_{n}(i)}\right)\right\|+\left\|\sum_{i=1}^{l_{n}} \lambda_{n}^{(i)} z_{\phi_{n}(i)}-z\right\|<\varepsilon,
\end{aligned}
$$

whenever $n \geq N_{3}(k, \varepsilon)$. This shows that $\left\|T^{k} z-z\right\|<\varepsilon$ for $k \geq N_{2}(\varepsilon)$.

Lemma 2.6. Suppose that $\left\{x_{n}\right\}_{n \geq 0}$ and $\left\{y_{n}\right\}_{n \geq 0}$ are almost-orbits of $T$. Then $\left\{\left\|x_{n}-y_{n}\right\|\right\}$ converges as $n \rightarrow \infty$.

Proof. Put $a_{n}=\sup _{m \geq 0}\left\|x_{n+m}-T^{m} x_{n}\right\|$ and $b_{n}=\sup _{m \geq 0}\left\|y_{n+m}-T^{m} y_{n}\right\|$ for $n \geq 0$. Then $a_{n} \rightarrow 0$ and $b_{n} \rightarrow 0$ as $n \rightarrow \infty$. By (1.2) for each $\varepsilon>0$ and each integer $n \geq 1$ there exists an integer $N(\varepsilon, n) \geq 1$ such that if $m \geq N(\varepsilon, n)$, then $\left\|T^{m} x_{n}-T^{m} y_{n}\right\|-\left\|x_{n}-y_{n}\right\|<\varepsilon$. Since

$$
\begin{aligned}
\left\|x_{n+m}-y_{n+m}\right\| & \leq\left\|x_{n+m}-T^{m} x_{n}\right\|+\left\|T^{m} x_{n}-T^{m} y_{n}\right\|+\left\|T^{m} y_{n}-y_{n+m}\right\| \\
& \leq a_{n}+b_{n}+\left\|x_{n}-y_{n}\right\|+\varepsilon
\end{aligned}
$$

for $m \geq N(\varepsilon, n)$, letting $m \rightarrow \infty$, and then $n \rightarrow \infty$ and $\varepsilon \downarrow 0$, we have

$$
\limsup _{m \rightarrow \infty}\left\|x_{m}-y_{m}\right\| \leq \liminf _{n \rightarrow \infty}\left\|x_{n}-y_{n}\right\|
$$

and so the conclusion holds.

Lemma 2.7. Suppose that $\left\{x_{j}^{(p)}\right\}_{j \geq 0}(p=1,2, \cdots)$ are almost-orbits of $T$ satisfying $\sup \left\{\left\|x_{j}^{(p)}\right\|: j \geq 0, p \geq 1\right\}<\infty$. Then for each $\varepsilon>0$ and each integer $n \geq 2$ there exist positive integers $N_{\varepsilon}$ and $i_{n}(\varepsilon)$, where $N_{\varepsilon}$ is independent of $n$, such that

$$
\left\|T^{k}\left(\sum_{p=1}^{n} \lambda_{p} x_{i}^{(p)}\right)-\sum_{p=1}^{n} \lambda_{p} T^{k} x_{i}^{(p)}\right\|<\varepsilon
$$

for all $k \geq N_{\varepsilon}, i \geq i_{n}(\varepsilon)$ and $\lambda \in \Delta^{n-1}$.

Proof. Take $f \in F(T)$ and set $K=\operatorname{clco}\left(\left\{x_{j}^{(p)}: j \geq 0, p \geq 1\right\} \cup\{f\}\right)$. For $\varepsilon>0$ take an integer $N_{\varepsilon} \geq 1$ and $\delta_{\varepsilon}>0$ in Lemma 2.3.

Since $\left\{\left\|x_{j}^{(p)}-x_{j}^{(q)}\right\|\right\}_{j \geq 0}$ converges as $j \rightarrow \infty$ by Lemma 2.6, for each $p, q$ there exists an integer $i_{0}(\varepsilon, p, q) \geq 1$ such that $\left\|x_{i}^{(p)}-x_{i}^{(q)}\right\|-\left\|x_{i+k}^{(p)}-x_{i+k}^{(q)}\right\|<\delta_{\varepsilon} / 3$ if $i \geq i_{0}(\varepsilon, p, q)$ and $k \geq 0$. Moreover there is an integer $i_{1}(\varepsilon, p) \geq 1$ such that $a_{i}^{(p)}<\delta_{\varepsilon} / 3$ for all $i \geq i_{1}(\varepsilon, p)$, where $a_{i}^{(p)}=\sup _{j \geq 0}\left\|x_{i+j}^{(p)}-T^{j} x_{i}^{(p)}\right\|$ for $i \geq 0$.

Put $i_{n}(\varepsilon)=\max \left\{i_{0}(\varepsilon, p, q), i_{1}(\varepsilon, p): 1 \leq p, q \leq n\right\}$. If $i \geq i_{n}(\varepsilon)$ and $k \geq N_{\varepsilon}$, then

$$
\begin{aligned}
& \left\|x_{i}^{(p)}-x_{i}^{(q)}\right\|-\left\|T^{k} x_{i}^{(p)}-T^{k} x_{i}^{(q)}\right\| \\
& \leq\left\|x_{i}^{(p)}-x_{i}^{(q)}\right\|-\left\|x_{i+k}^{(p)}-x_{i+k}^{(q)}\right\|+a_{i}^{(p)}+a_{i}^{(q)}<\delta_{\varepsilon}
\end{aligned}
$$

for $1 \leq p, q \leq n$ and so Lemma 2.3 gives the desired conclusion. 
By Lemma 2.6 an almost-orbit $\left\{x_{n}\right\}$ of $T$ is bounded, because of $F(T) \neq \phi$.

In what follows take $f \in F(T)$ and set $K=\operatorname{clco}\left(\left\{x_{i}: i \geq 0\right\} \cup\{f\}\right)$ for an almost-orbit $\left\{x_{n}\right\}$ of $T$.

Lemma 2.8. Suppose that $\left\{x_{i}\right\}_{i \geq 0}$ is an almost-orbit of $T$. Then for each $\varepsilon>0$ there exists an integer $N_{\varepsilon} \geq 1$ such that for each $k \geq N_{\varepsilon}$, there is an integer $N_{k, \varepsilon} \geq 1$ satisfying

$$
\frac{1}{n} \sum_{i=0}^{n-1} x_{i+l} \in F_{\varepsilon}\left(T^{k}\right) \text { for all } n \geq N_{k, \varepsilon} \text { and } l \geq 0 .
$$

Proof. Let $\varepsilon>0$. By Lemma 2.4 there exist an integer $N_{1}(\varepsilon) \geq 1$ and $\delta_{1}\left(=\delta_{1}(\varepsilon)\right)>$ 0 such that

$$
\operatorname{clco}\left(F_{\delta_{1}}\left(T^{k}\right) \cap K\right) \subset F_{\frac{\varepsilon}{3}}\left(T^{k}\right) \cap K
$$

for $k \geq N_{1}(\varepsilon)$. Let $\delta(=\delta(\varepsilon))=\min \left(\frac{\varepsilon}{12 D_{K}}, \delta_{1}\right)$.

Also, from (1.2) we can choose an integer $N_{2}(\varepsilon) \geq 1$ such that if $k \geq N_{2}(\varepsilon)$,

$$
\left\|T^{k} u-T^{k} v\right\|-\|u-v\|<\varepsilon / 3
$$

for all $u, v \in K$.

Moreover by Lemma 2.7 for any integer $p \geq 1$ there are positive integers $N_{3}(\varepsilon)$ and $i_{p}(\varepsilon)$, where $N_{3}(\varepsilon)$ is independent of $p$, such that

$$
\left\|T^{k}\left(\frac{1}{p} \sum_{j=0}^{p-1} x_{i+j+l}\right)-\frac{1}{p} \sum_{j=0}^{p-1} T^{k} x_{i+j+l}\right\|<\frac{\delta^{2}}{8}
$$

for $k \geq N_{3}(\varepsilon), i \geq i_{p}(\varepsilon)$ and $l \geq 0$.

Put $N_{\varepsilon}=\max \left\{N_{i}(\varepsilon): 1 \leq i \leq 3\right\}$ and fix $k \geq N_{\varepsilon}$.

Choose $p(=p(k, \varepsilon)) \geq 1$ so that $\frac{D_{K} k}{p} \leq \frac{\delta^{2}}{2}$. Since $\left\{x_{i}\right\}_{i \geq 0}$ is an almost-orbit of $T$, there exists an integer $N_{4}(\varepsilon) \geq 1$ such that $\sup _{q \geq 0}\left\|x_{m+q}-T^{q} x_{m}\right\|<\frac{\delta^{2}}{8}$ for $m \geq N_{4}(\varepsilon)$. Set $w_{i}=\frac{1}{p} \sum_{j=0}^{p-1} x_{i+j}$ for $i \geq 0$.

If $i \geq i_{p}(\varepsilon)+N_{4}(\varepsilon)$, by (2.13) we have

$$
\begin{aligned}
& \left\|w_{i+k+l}-T^{k} w_{i+l}\right\| \\
& \leq\left\|\frac{1}{p} \sum_{j=0}^{p-1}\left(x_{i+j+k+l}-T^{k} x_{i+j+l}\right)\right\|+\left\|\frac{1}{p} \sum_{j=0}^{p-1} T^{k} x_{i+j+l}-T^{k}\left(\frac{1}{p} \sum_{j=0}^{p-1} x_{i+j+l}\right)\right\|<\frac{\delta^{2}}{4}
\end{aligned}
$$

for all $l \geq 0$. We also have from (2.12)

$$
\begin{aligned}
\left\|w_{i+k}-T^{k} w_{i}\right\| & \leq\left\|w_{i+k}-f\right\|+\left\|T^{k} f-T^{k} w_{i}\right\| \\
& \leq\left\|w_{i+k}-f\right\|+\left\|f-w_{i}\right\|+\frac{\varepsilon}{3} \leq 2 D_{K}+\frac{\varepsilon}{3}:=D_{1}(\varepsilon)
\end{aligned}
$$

for $i \geq 0$. 
Choose $N_{5}(k, \varepsilon) \geq i_{p}(\varepsilon)+N_{4}(\varepsilon)+1$ such that $\frac{D_{1}(\varepsilon)\left\{i_{p}(\varepsilon)+N_{4}(\varepsilon)\right\}}{n}<\frac{\delta^{2}}{4}$ for all $n \geq N_{5}(k, \varepsilon)$. If $n \geq N_{5}(k, \varepsilon)$, it then follows from (2.14) and (2.15) that

$$
\begin{aligned}
& \frac{1}{n} \sum_{i=0}^{n-1}\left\|w_{i+l}-T^{k} w_{i+l}\right\| \\
& \leq \frac{1}{n} \sum_{i=0}^{n-1}\left\|w_{i+l}-w_{i+k+l}\right\|+\frac{1}{n}\left(\sum_{i=0}^{i_{p}+N-1}+\sum_{i=i_{p}+N}^{n-1}\right)\left\|w_{i+k+l}-T^{k} w_{i+l}\right\| \\
& \leq \frac{D_{K} k}{p}+\frac{\left(i_{p}+N\right) D_{1}(\varepsilon)}{n}+\frac{\delta^{2}}{4} \leq \delta^{2}
\end{aligned}
$$

for all $l \geq 0$, where $N=N_{4}(\varepsilon)$ and $i_{p}=i_{p}(\varepsilon)$. Finally, choose $N_{6}(k, \varepsilon) \geq 1$ so that $\frac{(p-1) D_{K}}{2 n}<\frac{\varepsilon}{12}$ for all $n \geq N_{6}(k, \varepsilon)$. Put $N_{k, \varepsilon}=\max \left(N_{5}(k, \varepsilon), N_{6}(k, \varepsilon)\right)$. Let $n \geq N_{k, \varepsilon}$ and $l \geq 0$.

Set $A(k, n, l)=\left\{i \in Z: 0 \leq i \leq n-1\right.$ and $\left.\left\|w_{i+l}-T^{k} w_{i+l}\right\|>\delta\right\}$ and $B(k, n, l)=$ $\{0,1, \cdots, n-1\} \backslash A(k, n, l)$.

By $(2.16), \sharp A(k, n, l) \leq n \delta$, where $\sharp$ denotes cardinality. Since $\delta D_{K}<\frac{\varepsilon}{12}$ and $\frac{(p-1) D_{K}}{2 n}<\frac{\varepsilon}{12}$, by $(2.11)$ we have

$$
\begin{aligned}
& \frac{1}{n} \sum_{i=0}^{n-1} x_{i+l}=\frac{1}{n} \sum_{i=0}^{n-1} w_{i+l}+\frac{1}{n p} \sum_{i=1}^{p-1}(p-i)\left(x_{i+l-1}-x_{i+l+n-1}\right) \\
& =\left[\frac{1}{n}(\sharp A(k, n, l)) \cdot f+\frac{1}{n} \sum_{i \in B(k, n, l)} w_{i+l}\right]+\left[\frac{1}{n} \sum_{i \in A(k, n, l)}\left(w_{i+l}-f\right)\right] \\
& +\frac{1}{n p} \sum_{i=1}^{p-1}(p-i)\left(x_{i+l-1}-x_{i+l+n-1}\right) \\
& \in \operatorname{clco}\left(F_{\delta}\left(T^{k}\right) \cap K\right)+B_{\frac{\varepsilon}{12}}+B_{\frac{\varepsilon}{12}} \subset\left(F_{\frac{\varepsilon}{3}}\left(T^{k}\right) \cap K\right)+B_{\frac{\varepsilon}{6}}
\end{aligned}
$$

for all $l \geq 0$. Combining this with (2.12) we find the desired claim.

Lemma 2.9. Suppose that the norm of $X$ is Fréchet differentiable and that $\left\{x_{n}\right\}$ is an almost-orbit of $T$. Then the following hold:

(i) $\left\{\left\langle x_{n}, J(f-g)\right\rangle\right\}$ converges as $n \rightarrow \infty$ for every $f, g \in F(T)$, where $J$ is the normalized duality map of $X$.

(ii) $F(T) \cap$ clco $\omega_{w}\left(\left\{x_{n}\right\}\right)$ is at most a singleton.

Proof. Let $\lambda \in(0,1)$ and $f, g \in F(T)$. By Lemma 2.7 and (1.2) for $\varepsilon>0$ there exist an integer $N_{\varepsilon} \geq 1$ and $i_{2}(\varepsilon) \geq 1$ such that if $k \geq N_{\varepsilon}$ and $n \geq i_{2}(\varepsilon)$,

$$
\begin{aligned}
& \left\|T^{k}\left(\lambda x_{n}+(1-\lambda) f\right)-\lambda T^{k} x_{n}-(1-\lambda) f\right\|<\varepsilon, \\
& \left\|T^{k} u-T^{k} v\right\|-\|u-v\|<\varepsilon
\end{aligned}
$$

for all $u, v \in K$. Then we have

$$
\begin{aligned}
& \left\|\lambda x_{n+m}+(1-\lambda) f-g\right\| \leq \lambda\left\|x_{n+m}-T^{m} x_{n}\right\| \\
& +\left\|\lambda T^{m} x_{n}+(1-\lambda) f-T^{m}\left(\lambda x_{n}+(1-\lambda) f\right)\right\|+\left\|\lambda x_{n}+(1-\lambda) f-g\right\|+\varepsilon \\
& \leq \sup _{l \geq 0}\left\|x_{n+l}-T^{l} x_{n}\right\|+\left\|\lambda x_{n}+(1-\lambda) f-g\right\|+2 \varepsilon
\end{aligned}
$$


for $m \geq N_{\varepsilon}$ and $n \geq i_{2}(\varepsilon)$. Letting $m \rightarrow \infty$, and then $n \rightarrow \infty$ and $\varepsilon \downarrow 0$, we get

$$
\limsup _{m \rightarrow \infty}\left\|\lambda x_{m}+(1-\lambda) f-g\right\| \leq \liminf _{n \rightarrow \infty}\left\|\lambda x_{n}+(1-\lambda) f-g\right\|
$$

and so $\left\|\lambda x_{n}+(1-\lambda) f-g\right\|$ converges as $n \rightarrow \infty$. The claim (ii) is shown by the same way as in [7, Lemma 3.6].

Using Lemmas 2.5, 2.8 and 2.9, by the same argument used in the proof of [8, Theorem] we can easily prove Theorem 1.1.

We also have the following result on the weak convergence of an almost-orbit $\left\{x_{n}\right\}$ of $T$.

Theorem 2.10. Let $\left\{x_{n}\right\}_{n \geq 0}$ be an almost-orbit of $T$. Suppose that

$$
\mathrm{w}-\lim _{n \rightarrow \infty}\left(x_{n}-x_{n+1}\right)=0 .
$$

Then we have $\omega_{w}\left(\left\{x_{n}\right\}\right) \subset F(T)$. Further if the norm of $X$ is Fréchet differentiable, $\left\{x_{n}\right\}$ converges weakly to an element in $F(T)$.

Proof. Let $u \in \omega_{w}\left(\left\{x_{n}\right\}\right)$. Then by definition there is a subsequence $\left\{p_{m}\right\}$ of $\{p\}$ such that $\mathrm{w}-\lim _{m \rightarrow \infty} x_{p_{m}}=u$. We have from assumption $\mathrm{w}-\lim _{m \rightarrow \infty} \frac{1}{n} \sum_{i=0}^{n-1} x_{i+p_{m}}$ $=\mathrm{w}-\lim _{m \rightarrow \infty} x_{p_{m}}=u$ for any fixed $n \geq 1$. Thus Lemmas 2.5 and 2.8 show $u \in F(T)$. The last assertion follows from Lemma 2.9 (ii).

Remark 2.1. (i) By using the same argument as in [9] with the aid of Lemmas 2.5, 2.7 and 2.8 we see that in Theorem 1.1 the assumption that the norm of $X$ is Fréchet differentiable may be replaced by the assumption that $X$ satisfies Opial's property.

(ii) We can also prove more general ergodic theorems corresponding to [10, Theorems 1 and 2] for semigroups of asymptotically nonexpansive mappings in the intermediate sense. As a consequence we see that [14, Corollary 4] holds for asymptotically nonexpansive mappings in the intermediate sense.

(iii) We do not know whether Theorem 2.10 is valid for asymptotically nonexpansive mappings satisfying (1.1).

\section{REFERENCES}

[1] J.B. BAILlon, Un théorème de type ergodique pour les contractions non linéaires dans un espace de Hilbert, C. R. Acad. Sci. Paris Sér. A 280 (1975), 1511-1514. MR 51:11205

[2] R.E. BRUCK, A simple proof of the mean ergodic theorem for nonlinear contractions in Banach spaces, Israel J. Math. 32 (1979), 107-116. MR 80j:47066

[3] R.E. BRUCK, On the convex approximation property and the asymptotic behavior of nonlinear contractions in Banach spaces, Israel J. Math. 38 (1981), 304-314. MR 82h:47051

[4] R.E. Bruck, T. Kuczumow And S. Reich, Convergence of iterates of asymptotically nonexpansive mappings in Banach spaces with the uniform Opial property, Colloq. Math. LXV (1993), 169-179. MR 94h:47106

[5] K. Goebel And W.A. Kirk, A fixed point theorem for asymptotically nonexpansive mappings, Proc. Amer. Math. Soc. 35 (1972), 171-174. MR 45:7552

[6] W.A. KIRK, Fixed point theorems for non-Lipschitzian mappings of asymptotically nonexpansive type, Israel J. Math. 17 (1974), 339-346. MR 49:11330

[7] I. MiYAdera AND K. KobAyASI, On the asymptotic behaviour of almost-orbits of nonlinear contraction semigroups in Banach spaces, Nonlinear Analysis 6 (1982), 349-365. MR 84c: 47064

[8] H. OKA, A nonlinear ergodic theorem for asymptotically nonexpansive mappings in Banach spaces, Proc. Japan Acad. 65 (1989), 284-287. MR 91c: 47115

[9] H. OKA, On the nonlinear mean ergodic theorems for asymptotically nonexpansive mappings in Banach spaces, Public. Res. Inst. Math. Sci. (Kokyûroku) 730 (1990), 1-20. 
[10] H. ОкA, Nonlinear ergodic theorems for commutative semigroups of asymptotically nonexpansive mappings, Nonlinear Analysis 18 (1992), 619-635. MR 93d:47126

[11] S. ReIch, Almost convergence and nonlinear ergodic theorems, J. Approx. Theory 24 (1978), 269-272. MR 80b:47079

[12] S. Reich, Nonlinear ergodic theory in Banach spaces, Argonne National Laboratory Report No.79-69 (1979).

[13] S. ReICH, Weak convergence theorems for nonexpansive mappings in Banach spaces, J. Math. Anal. Appl. 67 (1979), 274-276. MR 80d:47090

[14] S. ReIch AND H.K. XU, Nonlinear ergodic theory for semigroups of Lipschitzian mappings, Comm. Appl. Nonlinear Analysis 1 (1994), 47-60. MR 95k:47099

[15] K.K. TAN AND H.K. XU, The nonlinear ergodic theorem for asymptotically nonexpansive mappings in Banach space, Proc. Amer. Math. Soc. 114 (1992), 399-404. MR 92e:47100

[16] K.K. TAN AND H.K. XU, A nonlinear ergodic theorem for asymptotically nonexpansive mappings, Bull. Austral. Math. Soc. 45 (1992), 25-36. MR 93c: 47068

School of Education, Department of Mathematics, Waseda University, 1-6-1 NishiWASEda, Shinjuku-Ku, Tokyo 169-50, Japan

Current address: Faculty of Engineering, Ibaraki University, 12-1 Nakanarusawa 4 chome, Hitachi, Ibaraki 316, Japan

E-mail address: oka@base.ibaraki.ac.jp 